\title{
SHARP SPECTRAL ESTIMATES FOR PERIODIC MATRIX-VALUED JACOBI OPERATORS
}

\author{
ANTON KUTSENKO
}

\begin{abstract}
For the periodic matrix-valued Jacobi operator $J$ we obtain the estimate of the Lebesgue measure of the spectrum $|\sigma(J)| \leqslant 4 \min _{n} \operatorname{Tr}\left(a_{n} a_{n}^{*}\right)^{\frac{1}{2}}$, where $a_{n}$ are off-diagonal elements of $J$. Moreover estimates of width of spectral bands are obtained.
\end{abstract}

\section{INTRODUCTION}

We consider a self-adjoint matrix-valued Jacobi operator $J: \ell^{2}(\mathbb{Z})^{m} \rightarrow \ell^{2}(\mathbb{Z})^{m}$ given by

$$
(J y)_{n}=a_{n} y_{n+1}+b_{n} y_{n}+a_{n-1}^{*} y_{n-1}, \quad n \in \mathbb{Z}, \quad y_{n} \in \mathbb{C}^{m}, \quad y=\left(y_{n}\right)_{n \in \mathbb{Z}} \in \ell^{2}(\mathbb{Z})^{m},
$$

where $a_{n}$ and $b_{n}=b_{n}^{*}$ are $p$-periodic sequences of the complex $m \times m$ matrices. It is well known (see e.g. [CG], [CGR], [KKu], [KKu1]) that the spectrum of this operator $\sigma(J)=\sigma_{a c}(J) \cup$ $\sigma_{p}(J)$, where absolutely continuous part $\sigma_{a c}(J)$ is a union of finite number of intervals and $\sigma_{p}(J)$ consists of finite number of eigenvalues of infinite multiplicity. Note that if $\operatorname{det} a_{n} \neq 0$ for all $n=1, \ldots, p$, then $\sigma(J)=\sigma_{a c}(J)$ and $\sigma_{p}(J)=\emptyset$ always. The main goal of this paper is to obtain estimate of length of spectrum $\sigma(J)$. We don't know such estimates for the matrix-valued Jacobi operators.

Theorem 1.1. The Lebesgue measure of spectrum of $J$ satisfy the following estimate

$$
\operatorname{mes}(\sigma(J)) \leqslant 4 \min _{n} \operatorname{Tr}\left(a_{n} a_{n}^{*}\right)^{\frac{1}{2}} .
$$

Remark 1.Note that this estimate does not depend on period $p$ and coefficients $b_{n}$, i.e. changing $p$ and $b_{n}$ we can't sufficiently increase the length of spectrum of $J$. If $a_{n}=0$ for some $n \in \mathbb{Z}$, then this estimate is sharp.

2. $(m=1)$ For the scalar case $m=1$ we have the estimate (see e.g. [DS], [Ku], [KKr])

$$
\operatorname{mes}(\sigma(J)) \leqslant 4\left|a_{1} a_{2} \ldots a_{p}\right|^{\frac{1}{p}}
$$

We reach equality for the case of discrete Shrödinger operator $J^{0}$ with $a_{n}^{0}=1, b_{n}^{0}=0$. Estimate (1.2) is better than (1.3), since min $\left|a_{n}\right| \leqslant\left|a_{1} a_{2} \ldots a_{p}\right|^{\frac{1}{p}}$.

3. The result similar to (1.3) was obtained in [PR] for general non periodic scalar case $(m=1)$.

4. From the Proof of Theorem 1.1 we obtain estimates of spectral bands, see (2.9).

Example (sharpness). We construct the Jacobi matrix $J$ whose spectrum satisfy mes $(\sigma(J))=$ $4 \min _{n}\left(\operatorname{Tr} a_{n} a_{n}^{*}\right)^{\frac{1}{2}}$. Let $J$ be Jacobi matrix with elements $a_{n}=I_{m}(m \times m$ identical matrix $)$ and $b_{n}=\operatorname{diag}(4 k)_{k=1}^{m}$ for any $n$. Since all $a_{n}$ and $b_{n}$ are diagonal matrix, then $J$ is unitarily

Date: November 21, 2018.

1991 Mathematics Subject Classification. $81 \mathrm{Q} 10$ (34L40 47E05 47N50).

Key words and phrases. matrix-valued Jacobi operator, Jacobi matrix, spectral estimates, measure of spectrum. 
equivalent to the direct sum of scalar Jacobi operators. In our case this is the direct sum of shifted discrete Shrödinger operators $\oplus_{k=1}^{m}\left(J^{0}+4 k I\right)$ ( $I$ is identical operator). Then

$$
\sigma(J)=\bigcup_{k=1}^{m} \sigma\left(J^{0}+4 k I\right)=\bigcup_{k=1}^{m}[-2+4 k, 2+4 k]=[2,2+4 m],
$$

which gives us $\operatorname{mes}(\sigma(J))=4 m=4\left(\operatorname{Tr} a_{n} a_{n}^{*}\right)^{\frac{1}{2}}$.

\section{Proof of Theorem 1.1}

Without lost of generality we may assume that $\min _{n} \operatorname{Tr}\left(a_{n} a_{n}^{*}\right)^{\frac{1}{2}}=\operatorname{Tr}\left(a_{0} a_{0}^{*}\right)^{\frac{1}{2}}$ and $p \geqslant 3$. It is well known (see e.g. [KKu], [KKu1]) that $J$ is unitarily equivalent to the operator $\mathcal{J}=\int_{[0,2 \pi)}^{\oplus} K(x) d x$ acting in $\int_{[0,2 \pi)}^{\oplus} \mathcal{H} d x$, where $\mathcal{H}=\mathbb{C}^{p m}$ and $p m \times p m$ matrix $K(x)$ is given by

where

$$
K(x)=\left(\begin{array}{ccccc}
b_{1} & a_{1} & 0 & \ldots & e^{-i x} a_{0}^{*} \\
a_{1}^{*} & b_{2} & a_{2} & \ldots & 0 \\
0 & a_{2}^{*} & b_{3} & \ldots & 0 \\
\ldots & \ldots & \ldots & \ldots & \ldots \\
e^{i x} a_{0} & 0 & 0 & \ldots & b_{p}
\end{array}\right)=K_{0}+K_{1}(x)
$$

$$
K_{0}=\left(\begin{array}{ccccc}
b_{1} & a_{1} & 0 & \ldots & 0 \\
a_{1}^{*} & b_{2} & a_{2} & \ldots & 0 \\
0 & a_{2}^{*} & b_{3} & \ldots & 0 \\
\ldots & \ldots & \ldots & \ldots & \ldots \\
0 & 0 & 0 & \ldots & b_{p}
\end{array}\right), \quad K_{1}(x)=\left(\begin{array}{ccccc}
0 & 0 & 0 & \ldots & e^{-i x} a_{0}^{*} \\
0 & 0 & 0 & \ldots & 0 \\
0 & 0 & 0 & \ldots & 0 \\
\ldots & \ldots & \ldots & \ldots & \ldots \\
e^{i x} a_{0} & 0 & 0 & \ldots & 0
\end{array}\right)
$$

The spectrum $\sigma(J)$ is

$$
\sigma(J)=\bigcup_{x \in[0,2 \pi]} \sigma(K(x))
$$

From (2.4)-(2.5) we obtain

$$
K_{0}-\left|K_{1}\right| \leqslant K(x) \leqslant K_{0}+\left|K_{1}\right|, \quad x \in[0,2 \pi]
$$

where

$$
\left|K_{1}\right|=\left(K_{1} K_{1}^{*}\right)^{\frac{1}{2}}=\left(\begin{array}{ccccc}
\left(a_{0}^{*} a_{0}\right)^{\frac{1}{2}} & 0 & 0 & \ldots & 0 \\
0 & 0 & 0 & \ldots & 0 \\
0 & 0 & 0 & \ldots & 0 \\
\ldots & \ldots & \ldots & \ldots & \ldots \\
0 & 0 & 0 & \ldots & \left(a_{0} a_{0}^{*}\right)^{\frac{1}{2}}
\end{array}\right)
$$

does not depend on $x$. Let $\lambda_{1}(x) \leqslant \ldots \leqslant \lambda_{N}(x)$ be eigenvalues of $K(x)$ and let $\lambda_{1}^{ \pm} \leqslant \ldots \leqslant \lambda_{N}^{ \pm}$ be eigenvalues of $K_{0} \pm\left|K_{1}\right|$. Using (2.7) we obtain

$$
\lambda_{n}^{-} \leqslant \lambda_{n}(x) \leqslant \lambda_{n}^{+}, \quad x \in[0,2 \pi]
$$

which with (2.6) gives us

$$
\sigma(J)=\bigcup_{x \in[0,2 \pi]}\left\{\lambda_{n}(x)\right\}_{n=1}^{N} \subset \bigcup_{n=1}^{N}\left[\lambda_{n}^{-}, \lambda_{n}^{+}\right] .
$$


Then

$$
\operatorname{mes}(\sigma(J)) \leqslant \sum_{n=1}^{N}\left(\lambda_{n}^{+}-\lambda_{n}^{-}\right)=2 \operatorname{Tr}\left|K_{1}\right|=4 \operatorname{Tr}\left(a_{n} a_{n}^{*}\right)
$$

Acknowledgements. I would to express thanks to prof. E. Korotyaev for useful discussions and remarks. Also I want to thank prof. B. Simon for useful comments and refferences to the paper $[\mathrm{PR}]$.

Many thanks to Michael J. Gruber, who told me that instead of $\left\|a_{n}\right\|$ rank $a_{n}$ (which was in the first version of this paper) is better to use $\operatorname{Tr}\left(a_{n} a_{n}^{*}\right)^{\frac{1}{2}}$ in (1.2).

\section{REFERENCES}

[CG] Clark, S.; Gesztesy, F. On Weyl-Titchmarsh theory for singular finite difference Hamiltonian systems, J. Comput. Appl. Math., 171 (2004) 151184.

[CGR] Clark, S.; Gesztesy, F.; Renger, W. Trace formulas and Borg-type theorems for matrix-valued Jacobi and Dirac finite difference operators. J. Diff. Eq. 219 (2005), 144-182.

[DS] P. Deift, B. Simon. Almost periodic Schrödinger operators III. The absolutely continuous spectrum in one dimension. Commun. Math. Phys., 90, 389411 (1983).

$[\mathrm{K}] \quad$ Kato T. Perturbation Theory for Linear Operators. Springer (February 15, 1995).

$[\mathrm{Ku}]$ Kutsenko A. Estimates of Parameters for Conformal Mappings Related to a Periodic Jacobi Matrix. Journal of Mathematical Sciences, Volume 134, Number 4 / April 2006, Pages 2295-2304.

[KKr] Korotyaev, E.; Krasovsky, I. Spectral estimates for periodic Jacobi matrices, Commun. Math. Phys. 234(2003), 517-532.

[KKu] Korotyaev, E., Kutsenko, A. Lyapunov functions for periodic matrix-valued Jacobi operators, AMS translations Series 2, 225 (2008), 117-131.

[KKu1] Korotyaev, E., Kutsenko, A. Borg type uniqueness Theorems for periodic Jacobi operators with matrix valued coefficients. Proc. of the AMS, Volume 137, Number 6, June 2009, Pages 1989-1996.

[L] Y. Last. On the measure of gaps and spectra for discrete 1D Schrödinger operators. Commun. Math. Phys., 149, 347-360 (1992).

$[\mathrm{PR}]$ A. Poltoratski, C. Remling. Reflectionless Herglotz Functions and Jacobi Matrices, Commun. Math. Phys. Volume 288 Number 3(2009), 1007-1021.

[RS] M. Reed ; B. Simon. Methods of modern mathematical physics. IV. Analysis of operators. Academic Press, New York-London, 1978.

[S] B. Simon, Orthogonal polynomials on the unit circle, Part 1 and Part 2, AMS, Providence, RI, 2005.

[S1] B. Simon, Trace Ideals and Their Applications: Second Edition. Mathematical Surveys and Monographs vol. $120,2005$.

[Te] G. Teschl, Jacobi Operators and Completely Integrable Nonlinear Lattices, Mathematical Surveys and Monographs, vol. 72, AMS, Rhode Island, 2000.

[vM] P. van Moerbeke. The spectrum of Jacobi matrices. Invent. Math. 37 (1976), no. 1, 45-81.

Laboratoire de Mécanique Physique, UMr CNRS 5469, Université Bordeaux 1, Talence 33405, France, EMail KUCEnKoa@RAMbler.ru 